\title{
CONSUMER RELATED LEGAL ASPECTS OF ELECTRONIC COMMERCE: THE CASE OF DENMARK
}

\author{
Morten Falch, Anders Henten \& Knud Erik Skouby \\ Center for Tele-Information \\ Technical University of Denmark
}

\begin{abstract}
The general legal framework covering economic transactions was created long before the creation of electronic commerce. Therefore most regulation more or less explicitly assumes that goods have a physical appearance and that all contractual issues are settled either orally or by the use of paper. Electronic Commerce mediated via the telecommunications network has enabled electronic ordering, electronic purchase and electronic payments. In addition, digital goods such as software have no physical appearance and can be subject to electronic distribution. Not all parts of the existing legislation fit into this new situation. This creates a number of legal and regulatory problems which, if not resolved, may restrict further growth of electronic commerce. This paper deals with the consumer related aspects of this regulatory challenge.
\end{abstract}

\section{INTRODUCTION}

Commerce mediated via the telecommunication network poses a number of regulatory and legal consumer-related questions. In principle, electronic commerce is subject to the same regulation as any other type of commerce, but quite often the existing legal framework does not take all problems related to electronic commerce into account. These problems are especially related to changes in three areas:

- $\quad$ parties can communicate on-line without physical presence;

- $\quad$ paper as a medium for confirmation of a contractual relationship is substituted by electronic digital communication;

- geographical barriers to commercial relations tend to disappear.

These problems are mainly related to two different aspects of commerce: namely, marketing issues and contractual aspects of the transaction. Section 3 deals with marketing issues while contractual aspects including telepayments are addressed in section 4 .

As the Internet is the primary medium for consumers to access the market for electronic commerce, section two deals with the conditions for Internet access in Denmark. 


\section{INTERNET IN DENMARK}

Internet has become essential for accessing information. It has become the general medium for putting information at public disposal. In realisation of that fact, an official committee on the future of libraries in Denmark in November 1997 proposed that Internet access must be free at libraries. Denmark has a very developed library system with public libraries in all 275 municipalities (often more than one in each municipality).

In the autumn of 1997, 53\% of households in Denmark had a PC, according to a survey conducted by Gallup for the Ministry of Research, and $12 \%$ of households had Internet access (Denmark 1997b). The number of Internet hosts in Denmark in July 1997 was 26 per 1,000 inhabitants, compared, for example, with the Finnish figure of 66 hosts per 1,000 inhabitants (the highest in the world) and 15 per 1,000 inhabitants in the UK (see: http://www.nz.com). More recent figures indicate that the penetration of Internet among Danish households continues to grow, while the penetration of PCs seems to have stabilised (Danish Statistics 1998).

Access to the Internet in Denmark may thus be said to be fairly developed. However, the majority of the population still does not have domestic access, and access in public institutions such as libraries is considered as important.

Internet access in Denmark really took off in the latter part of 1996. Prior to that, the incumbent telecommunication operator in Denmark, Tele Denmark, tried to offer a separate system, Diatel, with no great success. As a result, this system primarily became a gateway to the Internet. Today, Internet is by far the major system for electronic trade.

The costs of accessing the Internet consist of two elements: 1) the fee to the operator of the telephony network and 2) the fee to the Internet IAP. The first is regulated on basis of the law on universal service. The second is unregulated.

Both of these two cost elements can be subdivided into fixed costs and traffic dependent costs. In the case of the fee to an Internet access provider, the traffic dependent cost element has been abolished in most cases during the past two years. Only the fixed tariffs remain. In the case of the telephone line connection, there are both fixed and traffic dependent tariffs.

In Denmark, both the fixed tariff elements related to telephone line connections (installation and subscription) and the tariff for local calls are relatively high. Using a basket of tariffs - as done by the OECD in tariff comparisons (OECD various) - telephone prices are comparatively low in Denmark. However, the tariffs that are important for Internet access are relatively high.

There has, therefore, been a discussion in Denmark on if and how to lower the telephone line costs of accessing the Internet. An obvious question is 
whether it is justifiable and technically feasible to have lower tariffs when using the telephone network for Internet communications, than when using it for traditional conversation. Tele Denmark (the universal service provider) has (until now) offered two ways of cheaper access to the Internet. The first one is a specific Internet offer (called Internet Stik, Internet plug) that can be advantageous if having a relatively large usage of Internet access. The other one is not specific to Internet and can also be used for longer traditional telephone conversations. It is called HyggeSnak (cosy talk) and gives users a lower tariff per minute if connected for a period of at least 10,20 or 30 minutes.

In December 1997, a departmental order was issued allowing Tele Denmark to increase its subscription fees if lowering its traffic dependent tariffs. For heavy users of Internet access (via telephone connections), this is an advantage. However, the Internet industry - and the community at large - in Denmark still does not feel that the Minister of Research has yet fulfilled her promise to resolve the problems of sufficiently cheap access to the actual information highway (Internet).

\section{MARKETING ISSUES}

\subsection{Identification of partners}

An Internet connection provides a relatively cheap opportunity to open a business. It demands some knowledge, but very limited economic resources to make your own homepage and sell goods and services on the Internet, which provides access to a global market place. The only way the consumer generally can identify the provider is by the Internet address and this address will not tell anything about the provider's physical location. Some types of addresses are international and do not even reveal the nationality of the owner of the Web-site. Thus, it might be quite difficult to trace a certain supplier, if services or goods received by the consumer do not live up to expectations. Moreover, it is quite easy for a supplier to hide behind changing Internet addresses if he wishes to do so. If a consumer uses a search engine to find a certain brand name, it will often be difficult to ensure that he has entered the original homepage representing the brand or whether it is a page set up by somebody else. Internet addresses do often include the brand name of the supplier, but there are examples of misuse, where somebody has used a famous brand name as part of their Internet address.

These problems are approached in the Distance Contracts Directive from EU (1997a) where it is stated that "Prior to the conclusion of any distance contract, the consumer must be provided with clear and comprehensible information concerning:

- the identity and possibly the address of the supplier;

- the characteristics of the goods or services and their price;

- delivery costs; 
- the arrangements for payment, delivery or performance;

- the existence of a right of withdrawal;

- the period for which the offer or the price remains valid and the minimum duration of the contract, where applicable;

- the cost of using the means of distance communication. "

In relation to these demands, the possibility for tracing a service provider through the Internet provider is an advantage compared to mail order. Furthermore, according to Danish legislation (The Administration of Justice Act (Retsplejeloven) §299), the Internet provider is obliged to disclose any written contract with the service provider.

These obligations can only be enforced on suppliers within the EU or Denmark respectively, and that represents a problem for a global market place. One way to solve this problem is to provide a certification service for Web-sites. An example of such a service is provided by AICPA (the American Institute of Certified Public Accountants). AICPA has in September 1997 announced CPA WebTrust ${ }^{\text {SM }}$ (AICPA 1997) as a seal of assurance for electronic commerce. CPA Web Trust is designed for assessing commerce criteria for standard business practises and controls over transaction integrity and information protection. Consumers doing transactions via certified web sites are thus ensured a minimum reliability of suppliers.

In principle, the problems of identification are similar to those related to, e.g. mail order services. The difference is the global coverage of the Internet including lacking well-established procedures for the protection of trademarks from misuse.

Identification can also be a problem for suppliers, who want to know their customers. Therefore, it is a key interest of both consumers and suppliers to establish 'safe' ordering and payment systems. This aspect is discussed in section 4 .

\subsection{Protection from aggressive marketing}

With the Internet, the costs of distributing marketing information are drastically reduced. Therefore, there is a risk of 'information pollution' - i.e. that consumers are burdened with enormous amounts of information that they have no interest in and never asked for. Furthermore, electronic commerce makes it easier to register consumer behaviour, identify potential customers for specific products and contact these customer by direct mail.

Two concepts are relevant in this context: electronic traces and push technologies such as cookies. 


\subsubsection{Electronic traces}

Every time a consumer connects to the Internet, he will leave an electronic trace of his behaviour. These traces can be recordings of credit card transactions or behaviour on a certain Web-site - e.g. the number of hits on a specific page. The Danish 'Lov om betalingskort mv.; nr. 811 af 12.09.1994' (The Payment Card Act) states in \$24, that only information on usage that is strictly necessary for implementation of payment transactions must be registered, and that this information must only be given to a third party when necessary for implementation of payment transactions. This regulation only covers information related to payment transactions. Other types of electronic traces are not covered by this regulation.

\subsubsection{Cookies}

A cookie is similar to an electronic trace but stored in a file located on the consumer's hard-disk. The cookie is usually filed automatically, without prior permission from the consumer, when he connects to certain Web-sites. The cookie contains information that will be activated next time the consumer connects to the same Web-site. Cookies contain information on general formats, etc. and can reduce the amount of data to be downloaded when the Web-site is visited again. But a cookie can also contain information on consumer behaviour and interests. This can be used, e.g. to select the goods that should be presented to the consumer first by sign-on and on what terms. The selection will usually be outside the consumer's control and may even be without his knowledge. Cookies can make Websites user-friendlier as the information most relevant for the consumer automatically pops up on the screen, but it can also be misused. If cookies enable the owner of a Web-site to identify the user, we are entering an area which might be included in the laws following the EU Privacy Directive from 1995 (EU 1995), which addresses the protection of individuals with regard to processing of personal data. The use of cookies is not covered by the Danish act on private registers. In this act the emphasis is on registers and it is stated that a register within a company or an organisation may only contain information on private, economic or personal relations if it forms a natural part of the provision of the service offered. This is one example of a Danish law that gives relatively good protection to consumers through their specificity, but is inadequate in a changed environment.

\section{CONTRACTUAL ASPECTS}

The major part of electronic commerce today involves transactions on the Internet. But this open network still provides poor security, making customers reluctant to trust and use electronic commerce. This makes security a key issue. Security and trust have, however, many aspects. Functions in electronic commerce such as ordering, referral, delivery or 
payment typically require different kinds of security. Furthermore, on the institutional side, a public library may have different needs from a bank.

Security services for electronic commerce have to address the main threats that the actors and the system implementing the business processes face. These threats are mainly related to authentication, authorisation, integrity, non-repudiation, availability, and confidentiality.

Technical solutions exist for many of the above issues and are mainly related to firewalls, encryption, identification and user authentication methods and are increasingly related to World Wide Web (WWW) activities. (WWW transactions can be secured at three different levels: above HTTP, at the HTTP level, or below HTTP.)

Security protocols like SSL - Secure Socket Layer, which is applicationindependent and Secure-HTTP - are already widely accepted on the Internet, but additional security systems are required in the specific context of electronic commerce applications, both for a stronger protection of privacy and economic transactions and for better performances. Here, the concept of electronic signature is a key issue.

\subsection{Electronic signature}

The signature is an essential tool for authentication in any kind of contractual relationship, and the lack of an electronic counterpart to the traditional paper-based signature is a major barrier for electronic transactions. It is possible to engage in transactional relationships based on mutual trust between the partners. This happens every day both in electronic and in 'normal' transactions (e.g. orders made by phone), but the availability of an electronic signature will create trust, reduce risks, and thereby reduce costs related to electronic transactions.

In Denmark, electronic information has not yet the same legal validity as information written on paper. This has been confirmed in a recent verdict. A private chemical company had delivered fake data on their discharge of waste water to the public authorities. The company was accused of forgery, but the court found the company not guilty as the information was delivered on a floppy disk and not on paper. This case illustrated for the general public that there is an urgent need for legislation on the validity of electronic information.

As a part of the Danish plans for the development of the Information Society, it was decided that Denmark should be one of the first countries with an act on digital signature. It was announced to be adopted by the parliament in February 1997 and the Danish Ministry of Research and Information Technology promoted a proposal on digital signature, which would imply a full recognition of electronic signatures as legal proof. A 
digital signature should be based on an asymmetric key encryption, administered by a number of private but publicly certified key centres.

However, the Ministry of Justice has been very reluctant towards an act that would recognise the electronic signature as having the same legal value as a signature on paper. The traditional signature is an integrated part of all contractual legislation and a full recognition of electronic signatures will have an impact on about 10,000 legally covered contexts. Therefore, it was decided to present to the parliament two different models for recognition of digital signatures.

1) A full recognition of digital signatures in line with a paper-based signature, unless it is explicitly decided otherwise.

2) A more limited recognition, where a digital signature is not recognised by law, unless the relevant ministry has decided that digital signature can be applicable in this specific context.

If model 1 is chosen and adopted before all 10,000 legally covered contexts are reviewed, it may create unforeseen problems in relation to certain specific laws. If model 2 is chosen, it may take several years before a full recognition of digital signatures is obtained.

A draft law has been presented for public hearing in mid February 1998, with a more than 18 month delay. The draft law seems in principle to follow the second model. It is suggested that an electronic signature must be issued with a certificate specifying the validity of the signature. It can for instance be specified that a signature only is valid for communication with public authorities, but not for payments. It will be the responsibility of the receiver to ensure that the signature is valid, but the key centre has the obligation to provide information on any restrictions connected to the signature.

The delay of the implementation of a law on digital signature implies that the Danish legislation will be surpassed by the implementation of the EUdirective on digital signature, which was planned to take place mid 1998 (EU1997b).

\subsection{Tele-payment}

One of the advantages of electronic commerce on the Internet is that a trade relation easily can be initiated between two partners from two different locations with no prior relationships. Full realisation of these potentials requires an efficient tele payment system, defined as an electronic payment system supporting economic transactions initiated on the tele network. Lack of adequate electronic payment systems has been a major barrier towards the development of a consumer market for electronic commerce.

The Internet is only a transport medium and in principle there is no difference between a payment transaction communicated via the Internet 
and a payment transaction initiated through a letter. There are, however, a number of problems with electronic payments related to all the security aspects mentioned above, i.e. trust, confidentiality, integrity, authentication and non-repudiation. The problems fall in two main categories: transaction related and privacy related problems.

To trust a payment system, parties engaged in economic transaction will demand, e.g. that the payment is released as planned and that no erroneous payment, misuse or un-called-for disclosure occur.

Privacy related problems concern, e.g. anonymity securing that the buyer's identity is not used in the payments, and non-traceability, securing that the buyer cannot be identified and, also, that two different payment transactions by the same buyer cannot be linked.

New payment systems have therefore been developed in order to overcome these barriers for Internet commerce. In payment systems there will always be a trade off between security, flexibility and costs. Therefore, different systems are developed serving different types of payments.

The emerging payment systems can be classified into three main groups, according to the traditional payment model they resemble. These are basically: the card, cheque, and cash models.

\subsubsection{Card-based systems}

Today most payment transactions on the Internet are made by way of credit cards. The consumer types in credit card details, and this gives the supplier the right to withdraw an agreed amount from the credit card account. There are a number of risks related to this type of payment transaction and many consumers are, therefore, reluctant to use this type of payments.

First, there is the possibility that others on the Internet read the message containing information on credit card details. The Internet is a conglomerate of many networks operated by many different operators and the consumer will not know through which networks a particular message is transmitted. The reality behind this risk is subject to debate, and claims are made that payment on the Internet is at least as safe as any other credit card transaction.

Second, payments can be initiated by anyone with knowledge of the details of a credit card, as no signature is required. This is a risk for both consumers and dealers. Consumers will have the risk of paying for goods or services others have ordered in their name, and of being over- or re-charged by unreliable dealers. Dealers may have trouble with false orders, where payments are rejected. 
Third, credit card payments involve transaction costs that are too high for payment of small amounts.

Card-based systems provide, however, possibilities for secure use of the existing card authorisation infrastructure. In the traditional non-electronic structure, the risk of the transaction is usually split between the merchant and the card issuer according to certain security rules for identification and authentication. Significant improvements to this model have been introduced, however, in some of the network based electronic developments of this model. This is the case, for instance, with SET (Secure Electronic Transaction protocol) developed by a consortium including VISA and MasterCard which provides secure transaction, identification and authentication of both seller and buyer - or other parties in a transaction based on a third party issued key-certificate. The SET system is considered very secure, but it is not anonymous and it is traceable, both aspects in relation to the bank involved. A further complication is that the full SET system is expensive and heavy to handle as a global system, for both the merchant and the consumer.

The most widespread security system for card-based payments today is the SSL standard (Secure Socket Layer). The standard is developed by Netscape but is also used by Microsoft. This system uses digital signatures and encryption for some parts of the payment transaction and has no certification requirements for providers. Major credit card companies recommend SSL until SET is fully implemented.

\subsubsection{Check based systems}

Cheque-based payment systems are based on the paper check model and, as such, carry a reference to the issuer to prove their validity. Here digital signatures are used for signing and endorsing the check, and digital certificates for authenticating the payer. The security of these systems depends on the encryption chosen; it is not anonymous and it is traceable for the bank.

\subsubsection{The cash model}

In traditional commerce, small amounts can be paid in cash. In electronic commerce an electronic purse has been developed to serve the need for socalled micro payments. The principle is that the consumer opens an account with a service provider from which small amounts can be withdrawn, as long as the balance is positive. This type of payment can be used for payment of, e.g. database services, where each transaction does not cost more than a few cents. The merchant is secured payment as money is available on the account at the payment service provider, and the risk of the consumer is limited to the amount available in his 'purse'. 
The cash model relies on electronic tokens, which may be authenticated independently of the issuer. To achieve this, self-authenticated tokens or tamper-proof hardware are usually adopted. This is the case of systems like Electronic Purses or Wallets consisting of a chip card which can be loaded with an amount of 'electronic money' by means of an Automatic Teller Machine under control of the issuing bank. Here the cardholder bank account is debited during the process of loading the Electronic Wallet. These systems are typically less secure, but anonymous and non-traceable.

At the European level, it is recommended by the European Monetary Institute to allow only financial institutions to offer a prepaid electronic purse. This recommendation is implemented in Danish law in act 375 of 22 of May 1996 on saving institutions and issuers of prepaid small-moneycards. This law is implemented in order to protect both consumers and retailers using the card. The law sets a maximum limit on the amount available in an electronic purse (1,000 DKK or approx. $125 \mathrm{ECU})$ and defines financial requirements for the issuers similar to those demanded for other financial institutions.

\subsection{Intellectual property rights}

An important question particular related to trade with digital products is intellectual property rights (IPRs). IPRs are important for the production of information, knowledge and intellectual products of all kinds. If there were no IPRs, intellectual production would be limited. However, IPRs can also be said to limit the access of the broader public to intellectual products. The problems relating to electronic storage and distribution of intellectual products may not in principal vary from other means of communication. However, for holders of IPRs, the danger of not being able to uphold their rights is clear. Electronic information products may be copied again and again without the IPRs being respected. This may, conversely, lead to a situation where IPR-holders become more cautious putting their products out to the public.

\subsection{Right to a cooling-off period}

In Denmark, Internet commerce is covered by the Canvassing Act. This implies that consumers enjoy the same protection as with door-to-door selling. Even when an electronic contract has been signed with a valid digital signature, the consumer enjoys the right to a cooling-off period and it is possible to cancel the transaction within a certain time limit. This protects consumers receiving products not having the expected quality. On the other hand it adds to the risk of the suppliers. Marketing via the Internet does not give the supplier the same possibilities for selecting their customers as traditional canvassing do. 


\section{CONCLUSION}

The basic infrastructure for electronic commerce is in place even today. More than $50 \%$ of the private households have a PC and many of these are connected to the Internet. Both the number of PCs and the number of Internet connections are rapidly increasing. In addition to this Tele Denmark has announced an introduction of tele-shopping in combination with digital TV. This will be offered to all households subscribing to Tele Denmark's Cable-TV network. The service can be accessed via an ordinary TV connected to a set-top box that can be rented from Tele Denmark for a monthly rate of $4 £$.

Development of electronic commerce is however still hampered by a number of regulatory issues mainly related to payment and digital signature.

- Introduction of digital signatures increases security, but what happens if the signature is misused? This may occur if the consumer's personal code has been stolen. What is the liability of the consumer in this situation? In general it can be said that digital signatures will reduce the risks of abuse, but increase the liability of the consumer, compared to credit card transactions carried out without use of digital signatures.

- Validity of digital signatures shall be ensured by public key centres, but what will happen if nobody is willing to assume this responsibility?

- $\quad$ Another problem with providers of tele payments is control . From a technical point of view, it is very easy to set up a payment system. It is also possible to use a foreign service provided to handle payments related to domestic trade transactions, and consumers do not necessarily become aware of how their payments are handled. This problem is partly addressed with the SET structure of certification, which will provide some control with the providers of payment services, but it is still possible to use payment service providers hosted in countries with less tight control over financial institutions.

In Denmark the absolute dominant payment card is the national debit card the Dancard (Dankortet). This card is held by the great majority of consumers and can be used free of charge in almost any retail shop. The major barrier to a wider diffusion of commerce on the Internet is that payments with Dancard have not been implemented. The national payment service centre PBS (Pengeinstitutternes BetalingsService), which has an almost de facto monopoly on clearing of payment transactions in Denmark, has refused to handle Dancard transaction as long as they are not allowed to put a fee on the transaction. Therefore, an amendment to the Payment Card Act allowing such a fee is being discussed. A number of service providers including Danish Railways consider to use a foreign bank for administration of payments to circumvent this barrier.

In addition to these contractual issues, there is also a need to protect consumers through the regulation of marketing practises. It is necessary to 
ensure that companies using the Internet for commercial purposes can be identified and their physical location determined. In addition, a fair trading and marketing practice has to be ensured, e.g. through regulation of price labelling and registration of customers. In order to avoid information pollution the customer ought to be spared from receiving 'targeted' marketing material from a company in the future.

Electronic commerce is an international business and national regulations are very easy to circumvent. This is certainly an area where international regulation is necessary. It is difficult to implement international rules in an area where technology development is very dynamic, there are calls for flexible procedures, and which frequent updates of regulatory rules are allowed. However, such rules are necessary for a full realisation of the potential for electronic commerce.

\section{REFERENCES}

AICPA (1997) AICPA NewsFlash -9/16/97 - AICPA Launches CPA WebTrust Electronic Commerce Seal. http://www.aicpa.org/news/p091697a.html

Danish Statistics (1998) http://www.dst.dk/internet/k15/pcere.htm

Denmark (1997a) Ministry of Business and Industry: Forbrugerens retsbeskyttelse i grænseoverskridende digitale net, Copenhagen, Ministry of Business and Industry.

Denmark (1997b) Ministry of Research and Information Technology IT in Figures 1997, Ministry of Research and Information Technology, Copenhagen.

EU (1995) Directive 95/46EC of the European Parliament and of the Council of 24 October 1995 on the protection of individuals with regard to processing of personal data and on the free movement of such data.

EU (1997a) European Parliament and Council Directive 97/7/EC 17.02.1997 on the protection of consumers in respect of distance contracts.

EU (1997b) Communication from the Commission, Ensuring Security and Trust in Electronic Communication - Towards a European framework for digital signatures and encryption. $\operatorname{COM}(97) 503$ final.

OECD (various) Communications Outlook, OECD, Paris, various years. 